\title{
Assessment of nerve cathodal block for the percutaneous auricular vagus nerve stimulation
}

\author{
Amine M. Samoudi ${ }^{1}$, Tom Van de Steene ${ }^{1}$, Emmeric Tanghe ${ }^{1}$, Luc Martens ${ }^{1}$, and Wout Joseph ${ }^{1}$ \\ ${ }^{1}$ INTEC, Ghent University/imec, Ghent, Belgium, amine.samoudi@ugent.be
}

\begin{abstract}
Nerve cathodal block mechanism for the percutaneous auricular vagus nerve stimulation is investigated. The response of individual axons to stimulation will be assessed in terms of excitation, blocking and propagation of action potentials in order to optimize stimulation patterns. It was seen that the response obeyed the activating function remarkably well. The found sensitivity indices of the blocking threshold for variations in diameter and temperature $(61 \%$ and $15 \%$ respectively) are significantly higher than for the excitation threshold. Finally, the threshold needed for cathodal block (around $-5 \mathrm{~V}$ ) is far from the amplitudes used to stimulate the nerves (around $\mathbf{- 1} \mathrm{V}$ ) making cathodal block less likely to occur when stimulating with clinically used amplitudes in pVNS.
\end{abstract}

Keywords-Auricular branch of the vagus nerve; nerve block; Neuromodulation

\section{INTRODUCTION}

Stimulation of the cervical vagus nerve by implanted stimulation devices is gaining importance in the last decades as a treatment for major depression and refractory epilepsy, accelerated by the development of less invasive techniques to reduce the associated risks $[1,2]$. Those include the percutaneous stimulation of the auricular branch of the vagus nerve (pVNS). These nerve branches can be easily accessed by using needle electrodes in targeted regions of the auricle [2]. The therapeutic outcome is often depending on what fibers are activated during stimulation, thus it is important to know how axons at different locations, with respect to the electrode, having different geometries and diameters, react to several stimulation settings, mainly when increasing the stimulation amplitude. While different types of nerve blocking are already described in the literature for straight axons in a homogeneous medium [3, 4], this work will investigate the excitation/blocking behavior for axons in a realistic heterogeneous model of the auricle [2]. Besides the value and sensitivity of the blocking threshold itself, the response of the axons to supra-excitation-threshold stimuli in general will be studied as well.

\section{MATERIALS AND METHODS}

\section{A. Numerical model}

We used Sim4Life to perform numerical simulations of pVNS. Neural tissue models were combined with the low frequency solver. The Spatially Extended Nonlinear Node (SENN) model [5] was used to investigate transmembrane mechanisms. The numerical model consisted of a realistic high resolution model of a human ear (spatial resolution $3 \mathrm{~mm}$ ), 2 electrodes, and a spatial model of major blood vessels and nerves (Figure 1). Nerves were stimulated by cathodic single cycle mono-phasic pulse (amplitude $=1 \mathrm{~V}$, duration $1 \mathrm{~ms}$ ).

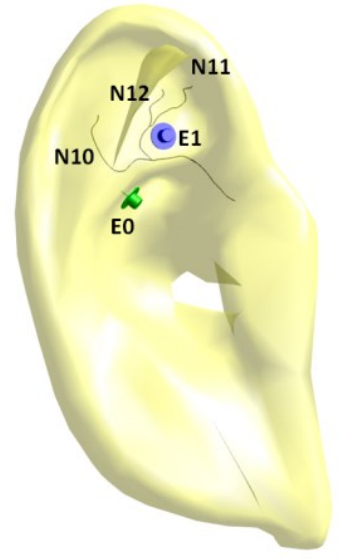

Figure 1: Model of the auricle including nerves, electrodes and vessels (not shown)

\section{B. Input and output parameters}

We consider the temperature and axon diameter to be the input parameters. These are each, separately, varied in their corresponding range as defined by [2]: the diameter varies from $7 \mu \mathrm{m}$ to $12 \mu \mathrm{m}$ in steps of $0.5 \mu \mathrm{m}$ and the temperature varies from $35.6{ }^{\circ} \mathrm{C}$ to $37.0{ }^{\circ} \mathrm{C}$ in steps of $0.2{ }^{\circ} \mathrm{C}$. Only one input variable is changed at a time. For the other, non-varying variables, the default value is used, which is $8 \mu \mathrm{m}$ for the diameter, $36.3^{\circ} \mathrm{C}$ for the temperature.

The first output variable of interest is the value of the blocking threshold at which the clear transition occurs from propagation to no propagation of an action potential along the axon. From this primary output, the sensitivity index is calculated as defined by [2]. This allows us to summarize the trend of the blocking thresholds as a function of the input variable into a single value. We will also refer to the sensitivity index with sensitivity. The sensitivity index is calculated as in (1).

$$
\text { S.I. }=\frac{o_{\max }-o_{\min }}{o_{\max }}
$$

In (1), $O_{\max }$ and $O_{\min }$ stand for the maximum and minimum absolute value of the blocking threshold for the different values of the input variable. From the blocking threshold, also the blocking/excitation ratio will be calculated, which is the ratio of the respective thresholds. 


\section{RESULTS AND DISCUSSION}

Table I shows the amplitudes needed to bloc nerve conduction and the different sensitivity analysis based on temperature and axon diameter variation. N11 and N12 show a very regular response: one action potential is generated at the excitation threshold at a location in accordance with the activating function; it keeps propagating normally at increasing amplitudes until the amplitude reaches the blocking threshold which is consistently a factor $6-10$ higher than the excitation threshold. Blocking is observed at different amplitudes as well. The rule of thumb blocking threshold $=8 *$ excitation threshold was valid for the individual action potentials. For N10, a more irregular response is seen. Action potentials are generated for different amplitudes at different locations along the nerve were the activating function is often positive, but not in all cases, thus no conduction block was detected for this axon. For this axon, no clear, sharp transition is seen between action potential propagation and blocking. In general, the response is in good correspondence with the activating function, both qualitatively and quantitatively to a certain extent as well. An important remark is that only for two axons, corresponding to those with the most regular response, the amplitude is within a realistic range for use in practice.

When increasing the temperature, the blocking threshold decreases, resulting in a mean S.I. of $15 \%$. This is significantly more than the mean S.I. of the excitation threshold of $0.8 \%$ [2]. The sensitivities of the blocking threshold for varying temperature are summarized in Table I as well. For all axons, a decreasing trend was seen for increasing diameter. The sensitivity for diameter variations is four times higher than for temperature variations, with a mean value of $61 \%$, which means that the blocking threshold becomes 2.5 times smaller within the defined diameter range. Similarly to the results for temperature variations, the sensitivities for the blocking thresholds for diameter variations are higher than for the excitation thresholds for which a mean sensitivity of $14 \%$ was established by [2]. A high sensitivity for diameter variations might be a desirable feature, because a certain range of fiber diameters can be targeted during stimulation by blocking other fibers. This behavior is visualized in figure 2, depending on its location, an axon will be either blocked (close to electrode), excited (second closest region) or nothing will happen (if far enough away from electrode). In figure 2 , the dependency on diameter is taken into account as well. Results also show that the threshold needed for cathodal block (around $-5 \mathrm{~V}$ ) is far from the amplitudes used to stimulate the nerves (around $1 \mathrm{~V}$ ) [2]. Thus cathodal block is less likely to occur when stimulating with clinically used amplitudes in pVNS.

\section{CONCLUSION}

A realistic numerical model for $p$ VNS application at the human auricle including vessels and nerves was used to investigate the cathodal block mechanism of the axons with respect to stimulation amplitude, temperature and axon diameter. Results show that the sensitivity of the blocking
TABLE I

SENSITIVITIES OF BLOCKING THRESHOLD FOR VARIATIONS IN TEMPERATURE AND DIAMETER

\begin{tabular}{cccc}
\hline \hline Axon & $\begin{array}{c}\text { S.I. of the mean } \\
\text { curve for } \\
\text { temperature (\%) }\end{array}$ & $\begin{array}{c}\text { S.I. of the mean } \\
\text { curve for diameter } \\
\text { dependency (\%) }\end{array}$ & $\begin{array}{c}\text { Blocking } \\
\text { threshold at } \\
\text { standard } \\
\text { conditions (V) }\end{array}$ \\
\hline N11 & 16.3 & 39.7 & -4.8 \\
N12 & 25.5 & 54.0 & -4.9 \\
\hline The & standard conditions for the last column are defined as a
\end{tabular}
temperature of $36.3{ }^{\circ} \mathrm{C}$, a diameter of $8 \mu \mathrm{m}$ and monophasic stimulation and no axon translation or shift of node locations.

threshold for changes in axon diameter and temperature $(61 \%$ and $15 \%$ respectively) are significantly higher than for the excitation threshold, the blocking threshold decreases for both increasing temperature and increasing diameter. Finally, cathodal block is less likely to occur at clinically used amplitudes in pVNS

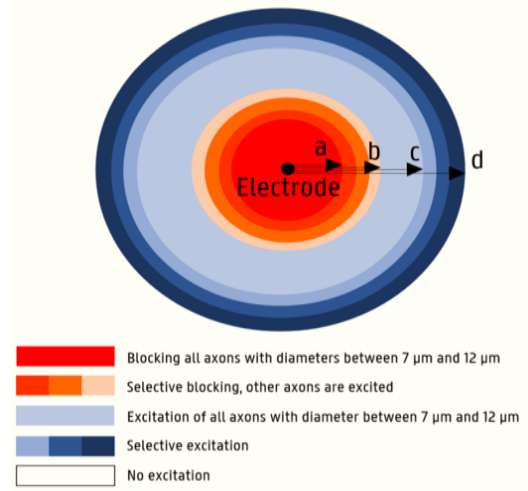

Figure 2: Expected behavior as a function of diameter for a certain amplitude, depending on the location of the axon with respect to the electrode

\section{ACKNOWLEDGMENT}

The research was supported by COST Action BM1309 (COST EMF-MED) and the FWO G003415N project. E. Tanghe is a postdoctoral fellow of the Research FoundationFlanders (FWO-V).

\section{REFERENCES}

[1] D.A. Groves, V.J. Brown. Vagal nerve stimulation: a review of its applications and potential mechanisms that mediate its clinical effects. Neurosci Biobehav Rev, 2009; 29: 493- 500.

[2] A. M. Samoudi, S. Kampusch, E. Tanghe, et al. Numerical Modelling of Percutaneous Auricular Vagus Nerve Stimulation: A Realistic 3D Model to Evaluate Sensitivity of Neural Activation to Electrode Position. Med Biol Eng Comput 2017;55:1763-1772.

[3] C. Tai, J. R. Roppolo, and W.C. de Groat. Analysis of nerve conduction block induced by direct current. J Comput Neurosci 2009;27(2).

[4] F. Rattay, Ways to approximate current-distance relations for electrically stimulated fibers. J. Theor. Biol. 1987;125(3), 339-349.

[5] J. P. Reilly, V. T. Freeman, and W. D. Larkin. Sensory effects of transient electrical stimulation - evaluation with a neuroelectric model. IEEE transactions on biomedical engineering 1985;12:1001-1011. 